\title{
Minimising Haemorrhagic Complications with Bariatric Surgery
}

\author{
Kamal K Mahawar ${ }^{1}$
}

Published online: 16 November 2015

(C) Springer Science+Business Media New York 2015

\section{Dear Editor,}

I read this paper [1] by Sroka and colleagues with huge interest. Haemorrhage is a recognised complication of bariatric surgery and one of the most frustrating. Aside from being a catastrophe itself, it can compromise healing of staple lines/ anastomoses or result in abscesses with attendant risk of late staple line/anastomotic leaks.

In my personal bariatric practice, I had an approximately $2 \%$ risk of serious haemorrhage requiring reoperation and each time it happened, I used to find my inability to anticipate it enormously depressing. After some reflection, I made two significant changes to my practice. First, I started asking anaesthetists to ensure that patient's blood pressure was close to his/her normal (at least $120 \mathrm{mmHg}$ ) systolic blood pressure before I finished. The second change I made was to spend an additional couple of minutes meticulously looking for and applying Liga clips to all suspected bleeding points (using the time when anaesthetist is attending to the blood pressure) until the returning irrigation fluid was completely clear. As a result of these changes, I have not had any return to theatre for haemorrhage in my last 400 odd bariatric operations.

I was pleasantly surprised on a visit to Dillemans' centre in Bruge, Belgium (with my anaesthetic colleague) recently, to find that he had independently reached similar conclusions and was also routinely elevating blood pressure at the end. Following the visit, I noticed much less resistance from my anaesthetic colleagues in raising the blood pressure for me even though I did not have any published evidence to back this request.

Kamal K Mahawar

kamal_mahawar@hotmail.com

1 Bariatric Unit, Sunderland Royal Hospital, Sunderland SR4 7TP, UK
It was hence interesting to see that authors of this study felt elevating blood pressure was such a significant variable that they actually controlled it across the three groups. They base it on their observation that patients had a much higher systolic blood pressure in recovery $(150-160 \mathrm{mmHg})$ when compared to the blood pressure at the end of the procedure $(90-100 \mathrm{mmHg})$ in their earlier cohort. Significantly, this reduced their reoperation rate for haemorrhage to zero from $3.0 \%$ (similar to my own experience). Even though this is not a direct conclusion from the randomised study (but a comparison of the entire randomised population with historic controls, not included in this study), this is probably the most fruitful finding of this paper.

Interestingly, as authors themselves mention, there is no trial directly addressing this point, and given the potentially huge impact confirmation of this observation may have in reducing haemorrhagic complications after not only bariatric surgery but also other types of laparoscopic and open surgery, I wonder if the potential risks (of additional drugs and elevating blood pressure) and benefits (in reducing haemorrhagic complications) should now be examined in a trial setting.

\section{Compliance with Ethical Standards}

Conflict of Interest The author declares no competing interests.

Statement on Human and Animal Rights This article does not contain any studies with human participants or animals performed by any of the authors.

\section{References}

1. Sroka G, Milevski D, Shteinberg D, et al. Minimizing hemorrhagic complications in laparoscopic sleeve gastrectomy - a randomized controlled trial. Obes Surg. 2015;25(9):1577-83. 